\title{
Stacked pin Devices for Imaging Applications
}

\author{
P. Louro ${ }^{1,2}$, A. Fantoni ${ }^{1}$, M. Fernandes ${ }^{1}$, M. Vieira ${ }^{1,2}$ \\ ${ }^{1}$ Electronics Telecommunication and Computer Dept. ISEL, \\ R.Conselheiro Emídio Navarro, 1949-014 Lisboa, Portugal \\ e-mail:plouro@deetc.isel.ipl.pt \\ ${ }^{2}$ CTS-UNINOVA' Quinta da Torre, 2829-516 Caparica, Portugal.
}

\begin{abstract}
In this paper we present results on the optimization of device architectures for colour and imaging applications, using a device with a TCO/pinpi'n/TCO configuration.

The effect of the applied voltage on the color selectivity is discussed. Results show that the spectral response curves demonstrate rather good separation between the red, green and blue basic colors. Combining the information obtained under positive and negative applied bias a colour image is acquired without colour filters or pixel architecture. A low level image processing algorithm is used for the colour image reconstruction.
\end{abstract}

Keywords- Optical devices, a-SiC heterostructures, colour sensors.

\section{INTRODUCTION}

The use of multilayered structures based on a-SiC:H alloys as color sensors has been an important topic of research in the field of sensing applications $[1,2,3]$. In these multilayered devices the light filtering is achieved through the use of different band gap materials, namely a$\mathrm{Si}_{1-\mathrm{x}} \mathrm{C}_{\mathrm{x}}: \mathrm{H}$. In these devices the spectral sensitivity in the visible range is controlled by the external applied voltage. Thus, proper tuning of the device sensitivity along the visible spectrum allows the recognition of the absorbed light wavelength, and consequently the identification of the RGB components of a coloured image [4].

In this paper color pinpi'n sensitive detectors are tested using the laser scanned photodiode technique (LSP) [5]. This technique allows a complete color analysis to be performed with a single two terminal detector element and an optically addressed readout technique. With this technique the image to acquire is optically mapped onto the sensing photodiode and a low-power light spot scans the device by the opposite side. The photocurrent generated by the moving spot is recorded as the image signal, and its magnitude depends on the light pattern localization and intensity. For image color acquisition the device is biased at different voltage values, which modulates the output image signal and allows the reconstruction of the color image $[6,7]$.

\section{EXPERIMENTAL DETAIL}

\section{A. Device architecture}

A series of stacked devices are analyzed in this work. The front $\mathrm{p}$-i-n structure is common to all devices while the back one has an intrinsic absorber layer which thickness was varied in order to optimize the device. The geometry of the stacked sensors is sketched in Figure 1.

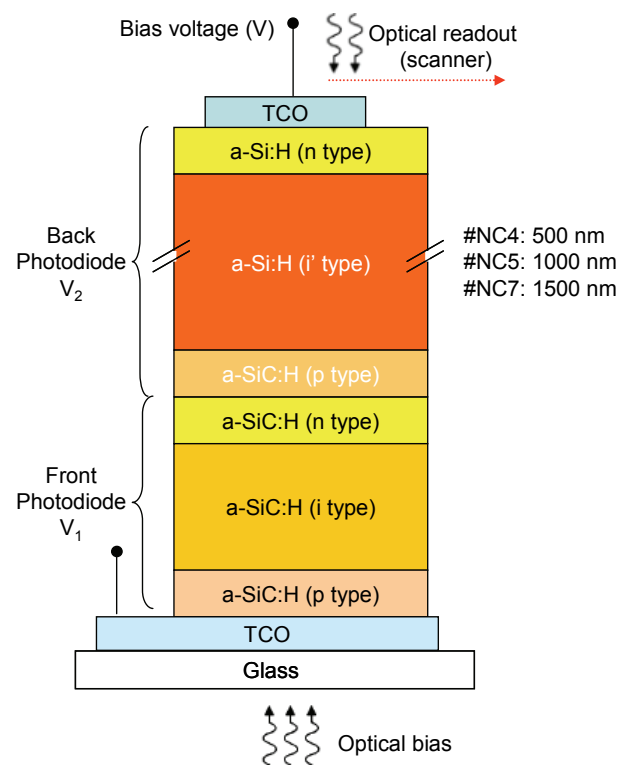

Figure 1 - Configuration of stacked structures.

The sensor is a double heterostructure device and consists of a glass/ITO/p-i-n a-SiC:H photodiode which faces the incident illumination, followed by a-SiC:H(-p) /Si:H(i')/SiC:H (-n')/ITO heterostructure, that allows the optical readout. The doped layers are based on a-SiC:H to increase image resolution and to prevent image blurring. To profit from the light filtering properties of the active absorbers, the intrinsic layer of the front diode is thinner and based on $\mathrm{a}-\mathrm{SiC}: \mathrm{H}$ and the back one is thicker and based on a-Si:H.

In the polychromatic operation mode different sensitivity ranges are selected by using a voltage scan 
waveform, and the photocurrent generated by the optical scanner measured to sample the image signals. This approach leads to different collection regions resulting in voltage controlled multispectral photodiodes, coding for red $(\mathrm{R})$, blue $(\mathrm{B})$, and green $(\mathrm{G})$ components.

\section{RESULTS AND DISCUSSION}

\section{A. Device operation}

In Figure 2 it is displayed the simulated generation/recombination profile within device \#NC7 for different wavelengths of the incident light at short circuit conditions. Typical values of band tail and gap state parameters for amorphous material were used. The doping level was adjusted in order to obtain approximately the same conductivity of the individual layers. In the a-SiC:H film the optical band gap of $2.1 \mathrm{eV}$ was chosen in compliance with the obtained experimental values.

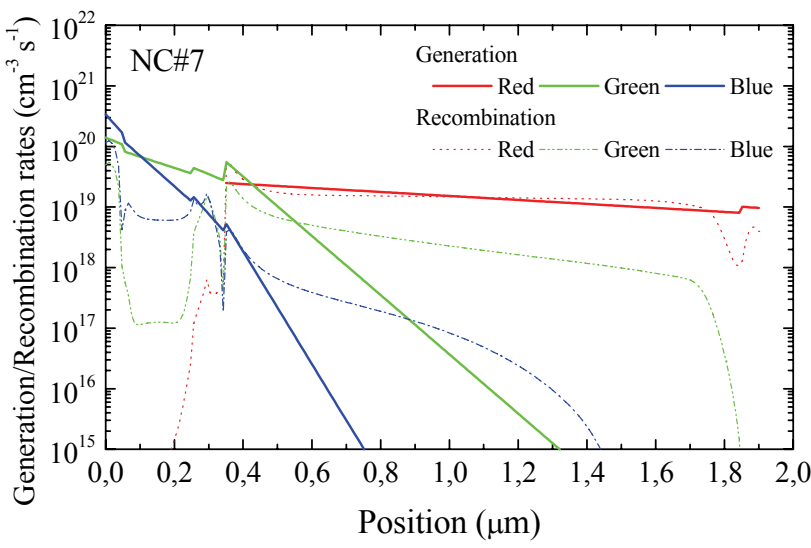

Figure 2 - Numerical simulation of the generation and recombination rates profiles under different light wavelengths in short-circuit condition within device \#NC7.

Results show that the light penetration depth controls the generation of carriers accordingly to the nature of the incident light. The thin a-SiC:H front absorber prevents the red light to generate carriers along the front photodiode. The generated photo carriers in this absorber arise from higher energy light photons (in the green/blue spectral range). The generation due to blue photons is dominant near the front $\mathrm{p}$ - $\mathrm{i}$ interface, which is due to the reduced penetration depth of the blue light caused by the higher value of the absorption coefficient in this spectral range.

\section{B. $\quad$ Voltage controlled sensitivity}

In Figure 3 it is displayed the collection efficiency, in the voltage range of $-6 \mathrm{~V}$ up to $+6 \mathrm{~V}$, under different steady state illumination conditions $\left(\lambda_{\mathrm{L}}=650 \mathrm{~nm}, 550 \mathrm{~nm}\right.$ and $450 \mathrm{~nm})$ for a red probe pulsed light $\left(\lambda_{\mathrm{S}}=650 \mathrm{~nm}\right)$.

Results show that the collection efficiency, at $650 \mathrm{~nm}$ depends on the optical bias conditions and on the thickness of the back intrinsic layer. The trend under illumination is different from the one observed in dark. When the applied bias changes from reverse to forward bias the carrier collection decreases, with different slopes in dark and under illumination, leading to voltage controlled color discrimination.

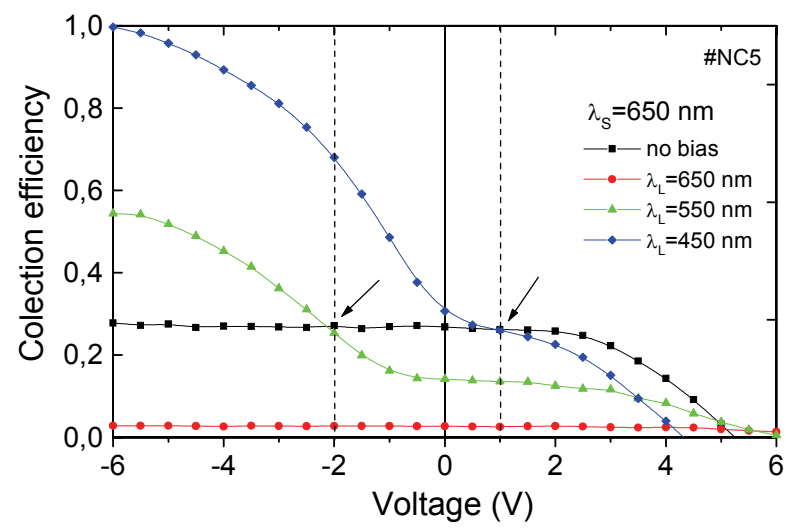

Figure 3 - Collection efficiency in dark and under red, green and blue steady state illumination using a red scanner $(\lambda \mathrm{s}=650 \mathrm{~nm})$.

The elimination of the green/blue signals at different voltage values is the key to enable colour sensing properties to these devices. By tuning the voltage to the value where the green signal is suppressed, the measured signal results only from contributions of the red and blue components, whereas that at $+1 \mathrm{~V}$ the blue signal is eliminated and the measured signal results only from contributions of the red and green components. At any reverse voltage value, the measured signal results from the contribution of the three components. Combining the information obtained at three applied voltages a colour image can be acquired. Thus, the recognition of the fundamental colours (red, green and blue) is possible through the correct selection of the applied voltage values. In device \#NC7 the colour separation is not as good as in sensor \#NC5 because when the green signal is suppressed the blue signal exhibits low amplitude. A similar situation occurs when the blue signal is suppressed, as at $+1 \mathrm{~V}$ the green signal is still low in amplitude. Thus, the configuration of sensor \#NC5 with a thinner absorber layer allows better separation of the fundamental colours.

\section{Image acquisition and reconstruction}

Figure 4a) shows the optical image of a graded wavelength mask (rainbow) that simulates the visible spectrum in the range between 400 and $700 \mathrm{~nm}$. This image was created using a Matlab program that computes the digital image. The picture was then printed in a transparent paper using the subtractive colour system of the printers. This optical image was projected onto the a$\mathrm{SiC}: \mathrm{H}$ front diode and acquired through the a-Si:H back one with a moving red scanner. For image acquisition two applied voltages were used to sample the image signal: + $1 \mathrm{~V}$ and $-6 \mathrm{~V}$. In Figure $4 \mathrm{~b}$ ) it is displayed the 2-D colour image reconstruction using the acquired image signals. The algorithm used for image color reconstruction took into account that at $-6 \mathrm{~V}$ the positive signals correspond to the blue/green contribution and the negative ones to the red inputs. The green information was extracted from the image signal sampled at $+2 \mathrm{~V}$, where the blue signal is 
almost suppressed (Figure 4b) and the green and red signals are negative.

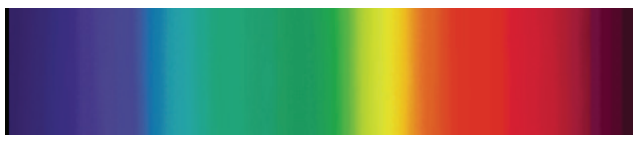

a)

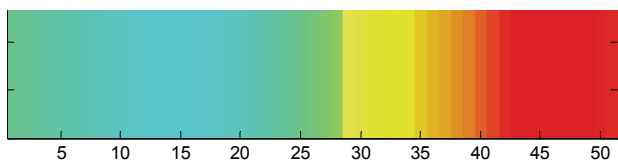

Figure 4 - a) Optical image projected onto the device and b) 2-D colour image reconstruction.

The combined integration of this information allows the fulfillment of the R, G and B channels. A good agreement between the optical image and the electrical reconstructed color image was obtained, although further optimization of the sampling threshold voltage values seems to be needed in order to improve the R, G and $B$ channels distributions. The device was then further analysed using as optical bias the light from the monochromator (in the $400 \mathrm{~nm}$ to $800 \mathrm{~nm}$ ) which corresponds to single wavelengths and the optical image of the printed rainbow mask where the colours result from multiple wavelength combinations. Under these conditions, the image signal was then measured. Results are plotted in Figure 5 and show that under these different optical biasing conditions the photocurrent of the device exhibits different trends. With monochromatic light (Figure 5a) the photocurrent exhibits a maximum around $465 \mathrm{~nm}$, then decreases for longer wavelengths, and reverses in sign around $550 \mathrm{~nm}$.

In the blue range the photocurrent is always positive, increasing as the reverse voltage increases. In the reddish part of the spectrum, the photocurrent reverses sign and its magnitude is independent on the applied voltage bias. When compared with the signal without light impinging the front diode (dark level at $750 \mathrm{~nm}$ ) at short circuit conditions, the red and blue signals have opposite signs and the green signal is almost suppressed, allowing blue and red recognition. The green information is obtained for the voltage bias that suppresses the blue signal, which occurs at slightly positive bias.

The analysis of the image signal obtained with the same device using as optical bias multiple wavelength combinations (Figure 5b) is similar to the one obtained with single wavelengths only in the reddish region of the visible spectrum, where in both cases the signal is reversed and independent on the applied bias. Main differences occur in the remaining regions of the spectrum, namely on the blue region where the signal exhibits sign reversal due to the presence of a red component in this part of the mask. This effect shows up also in the greenish region, as in this case the signal is similar to the one observed in the reddish part of the spectrum. Thus, we can conclude that with this mask all the colours result from a significant red component which influences the device photocurrent characteristics.

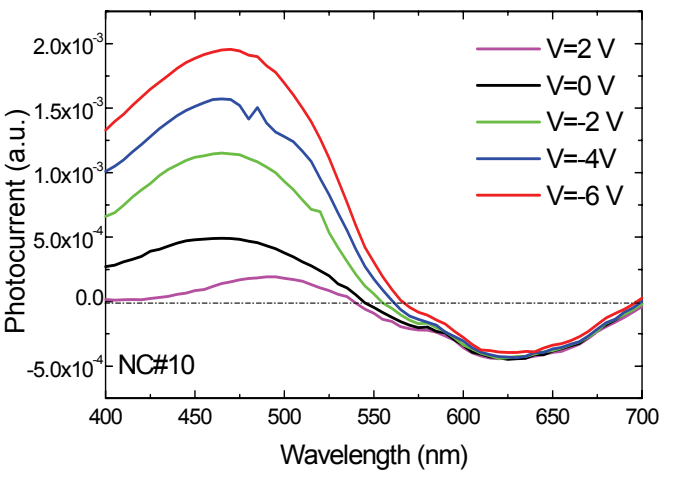

a)

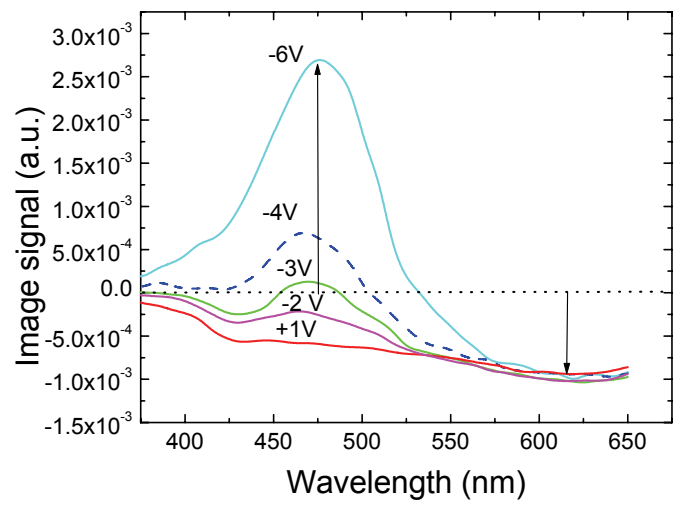

b)

Figure 5 - a) Photocurrent obtained with the light from the monochromator and b) Image signal obtained with the rainbow mask.

\section{Low level image processing}

Using these assumptions the RGB channels were fulfilled (Figure 6, solid lines) and the corresponding digital image representation reconstructed (Figure 7a).

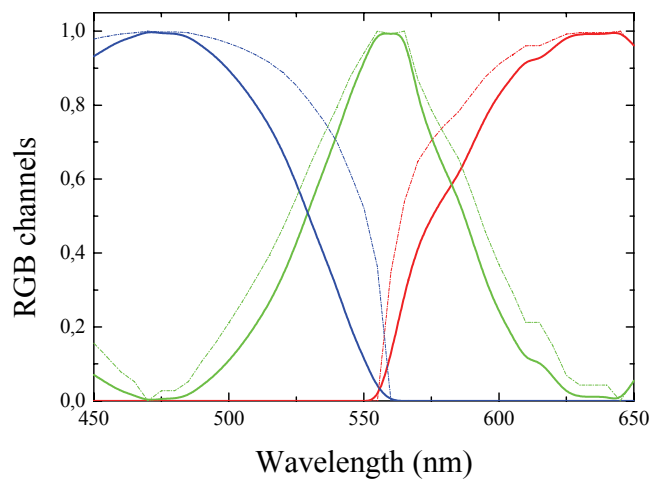

Figure 6 - Distribution of the Red, Green and blue channels (solid lines: without gamma correction; dashed lines: using different values of the coefficient for the gamma correction, $\gamma_{R}=0.5, \gamma_{G}=0.7, \gamma_{B}=0.3$ ).

Results show that the distribution is adequate, although not yet correctly balanced, as the region of intersection between the red and the green curves, as well as the overlap between the blue and the green channels occurs at moderate values of intensity. This makes difficult the representation of light cyan and light yellow/orange colours (Figure 7a). As already stated before the blue, green and red colours are adequately distributed along the spectrum, but the cyan, yellow and orange colours are missing, due to the fact that in the spectrum region where they should appear the RGB intensities are not high 
enough. Thus some further correction must be done in order to change the relative intensities of the channels. This can be done using the gamma correction procedure, which is a nonlinear operation used to code and decode luminance or tristimulus values in video or still images. In the simplest cases it is defined by the following power-law expression: $V_{\text {out }}=V_{\text {in }}^{\gamma}$, where the input $\left(V_{\text {in }}\right)$ and output values $\left(V_{\text {out }}\right)$ are non-negative real values, typically in a predetermined range such as 0 to 1 . The case $\gamma<1$ is often called gamma compression or gamma encoding and $\gamma>1$ is called gamma expansion or gamma decoding.

Gamma encoding helps to map data into a more perceptually uniform domain, so as to optimize perceptual performance of a limited signal range, such as a limited number of bits in each RGB component.

The use of the gamma correction using power coefficients less than one results in an enhanced increase of the image signal intensity in the region of low intensity. This effect grows with the decrease of the gamma power coefficient. Thus low values of $\gamma$ are responsible for an enhanced mapping of the original low intensity values. The gamma correction was then implemented, and the new distribution of the RGB channels is displayed in Figure $6 \mathrm{~b}$. The corresponding digital colour image representation obtained by using the gamma correction is displayed in Figure $7 b$.

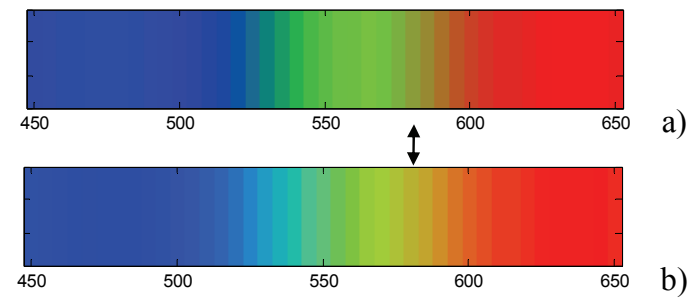

Figure 7 - Digital image representation of the rainbow picture acquired by the device: a) without gamma correction; b) with gamma correction $\left(\gamma_{\mathrm{R}}=0.5, \gamma_{\mathrm{G}}=0.7, \gamma_{\mathrm{B}}=0.3\right)$.

The use of the gamma correction results in a brighter image that allowed the representation of other colours that were missing in the original, uncorrected image. This was due mainly to use of different mapping of the channels intensities that allowed for the case of the cyan and yellow colours an overlap, respectively, between the blue and the green and the red and the green channels, at higher values of intensities (0.8 with gamma correction, and 0.5 without).

\section{CONCLUSIONS}

The various design parameters and image reconstruction algorithms are discussed. A physical model supported by electrical and numerical simulations give insight into the methodology used for image representation and color discrimination.

A low level image processing algorithm was used for the reconstruction of the colour image. Obtained results show that further optimization on the colour image reconstruction is necessary, as there is some mismatch in the reconstructed colour images. In order to advance more in this domain, some low level image processing algorithms were used to enhance the image intensity of each channel, which resulted in a better balanced coloured reconstructed image. These preliminary results based on the implementation of the gamma correction technique, show that with further image processing techniques better results can be achieved.

\section{REFERENCES}

[1] G. de Cesare, F. Irrera, F. Lemmi, F. Palma, IEEE Trans. on Electron Devices, Vol. 42, No. 5, May 1995, pp. 835-840.

[2] A. Zhu, S. Coors, B. Schneider, P. Rieve, M. Bohm, IEEE Trans. on Electron Devices, Vol. 45, No. 7, July 1998, pp. 1393-1398.

[3] M. Mulato, F. Lemmi, J. Ho, R. Lau, J. P. Lu, R. A. Street, J. of Appl. Phys., Vol. 90, No. 3 (2001), pp. 1589-1599.

[4] H. Stiebig, J. Gield, D. Knipp, P. Rieve, M. Bohm, Mat. Res. Soc. Symp. Proc 337 (1995) 815-821.

[5] M. Vieira, M. Fernandes, J. Martins, P. Louro, A. Maçarico, R. Schwarz, M. Schubert, IEEE Sensors Journal, 1 (2) (2001) 158167.

[6] P. Louro, M. Vieira, A. Fantoni, M. Fernandes, C. Nunes Carvalho, G. Lavareda, Sensors and Actuators A 123-124 (2005) 326-330.

[7] P. Louro, M. Vieira, Y. Vygranenko, M. Fernandes, A. Garção, Mat. Res. Soc. Symp. Proc., S. Francisco, 989 (2007) A12.04. 